\title{
A Post-Marketing Surveillance Study on the Effectiveness and Safety of Paliperidone ER among Filipino Adult Patients Diagnosed with Schizophrenia
}

\author{
Bernardo L. Conde, ${ }^{1}$ Michael P. Sionzon ${ }^{2,3}$ and Erwin G. Benedicto ${ }^{3}$ \\ ${ }^{1}$ Department of Neurology and Psychiatry, University of Sto. Tomas Hospital \\ ${ }^{2}$ Department of Psychiatry and Behavioral Medicine, College of Medicine and Philippine General Hospital, University of the Philippines Manila \\ 3Janssen Philippines
}

\begin{abstract}
Background. Schizophrenia affects 7 people per 1000 adult population worldwide and is a severe form of mental illness common in age group 15-35 year old. Paliperidone is the active metabolite of risperidone and was approved for treatment of schizophrenia in the Philippines by the Food and Drug Administration (FDA) in 2007. The drug has been shown to be safe and effective in clinical trials but no local study has investigated its effect among Filipino patients. Hence, the general objective of this study was to assess the safety and effectiveness of paliperidone ER among Filipino patients diagnosed with schizophrenia.
\end{abstract}

Methods. The study was a non-randomized, non-comparative, open-label trial involving adult patients seen at initial consult and at the end of study visit. Study duration was eight weeks and was conducted for three years as required by the FDA. The primary outcome for the study was overall severity of the illness at the initial visit and end visit (visit 2) using the Clinical Global Impression tool (CGI-S). This rating scale was used to rate the severity of a subject's psychotic condition on a 7-point scale ranging from 1 (not ill) to 7 (extremely severe).

Results. A total of 641 Filipino adults were enrolled in the study. Ninety one percent $(\mathrm{N}=586)$ had completed the follow-up into the second or end visit resulting in a 9\% drop out for the duration of the study. Follow-up period had an average of 58.05 ( $S D \pm 9.36$ ) days ranging from a minimum of 4 to a maximum of 98 days. Clinicians' assessment of the severity of illness showed that majority had shown improvement from their baseline clinical state with the use of Paliperidone ER. The proportion of severely ill had decreased by $15 \%$ while the propotion of those who were markedly ill had decreased by $35 \%$ by the end of the second visit. Overall, after eight weeks of paliperidone treatment, there was a decrease of $58 \%$ in the proportion of study participants who were assessed to be moderately to markedly ill at baseline and an increase of $74 \%$ in the proportion of subjects evaluated to be mildly ill, borderline mentally ill and normal at the end of the second visit. This means that there was a marked improvement in patients observed and reported

Corresponding author: Erwin G. Benedicto, MD

Johnson and Johnson Philippines

Edison Road, Barrio Ibayo 1700 Parañaque Philippines

Telephone: +632 8248935

Fax No: +632 7769819

Email: ebenedic@its.jnj.com symptoms, behavior and functions as assessed by physicians using CGI. Fifteen percent of study participants experienced at least one non-serious adverse event during the study. The five most common non-serious adverse events observed include sleeplessness, extrapyramidal symptoms described as Pseudo parkinsonian tremors, stiffness of body and DOB/EPS reaction hand tremors, depression, akathisia and dizziness.

Conclusion. Paliperidone ER administered at $6 \mathrm{mg}$ single dose improved symptoms and clinical signs among adult Filipinos diagnosed with schizophrenia who are moderately and markedly ill. The drug is well-tolerated, but the dose may need to be increased for more severely ill patients. Paliperidone ER is one of the current useful options for the treatment of patients with schizophrenia.

Key Words: schizophrenia, paliperidone, Filipino, safety, effectiveness

\section{Introduction}

Schizophrenia affects 7 people per 1000 adult population worldwide. It is a severe form of mental illness common in age group 15-35 year old and is considered to be one of the ten most debilitating diseases in the world. ${ }^{1,2}$ The disease accounts for $25 \%$ of psychiatric hospitalizations and $30 \%$ of those diagnosed will have deterioration of professional, social and emotional functions. ${ }^{3,4}$

Treatment modalities are available for schizophrenia but issues on the effectiveness of anti-psychotic drugs, including the recent second generations have been raised. ${ }^{5}$ Hence the search for new, more effective and safer alternatives still remain to be a goal of medical science.

Paliperidone was developed as a new therapeutic agent for the treatment of schizophrenia by Johnson \& Johnson Pharmaceutical Research \& Development, L.L.C. (J\&JPRD) in year 2006.6 It is the active metabolite of risperidone, a well-known second-generation antipsychotic drug. It was approved for treating schizophrenia worldwide in 2006 as paliperidone extended-release. ${ }^{7,8,9}$

Paliperidone (9-hydroxy-risperidone, R076477) is a selective, monoaminergic antagonist that exhibits the characteristic dopamine type 2 (D2) and serotonin (5hydroxytryptamine [5-HT]) type 2A (5HT2A) antagonism of the second-generation antipsychotic drugs. Unlike other antipsychotic drugs that usually recommend a dose titration 
to the maintenance dose, paliperidone has been developed with an extended-release (ER) formulation. This minimizes the risk of orthostatic hypotension and may lead to better efficacy and tolerability of the drug. Hence, the drug presents a unique pharmacological profile: single dosing, predominantly renal excretion and low drug-drug interaction risk. ${ }^{10,11}$

Previous studies have shown that paliperidone ER $(3,6,9,12$, or $15 \mathrm{mg} /$ day) was superior to placebo, as demonstrated by the decrease in positive and negative syndrome scale (PANSS) total scores from baseline to endpoint $(p=0.006)$. Paliperidone ER dose groups were also statistically significantly superior to placebo $(p=0.008)$ based on the mean change in the Personal and Social Performance (PSP) total score from baseline to endpoint. Moreover, paliperidone ER dose groups were superior to placebo based on the median change from baseline to endpoint in the Clinical Global Impression - Severity Scale (CGI-S) score. ${ }^{12,13}$

In August 2006, the Food and Drug Administration (FDA) approved paliperidone ER on a monitored release status. While paliperidone ER has been shown as safe and effective in previous trials, this study was conducted to further investigate its effect among Filipino patients as mandated. Hence, the general objective of this study was to assess the safety and effectiveness of paliperidone ER among Filipino patients diagnosed with schizophrenia.

\section{Methods}

The study was a non-randomized, non-comparative, open-label trial involving adult patients seen at initial consult (baseline) and at the end of study visit. Study duration was eight weeks and was conducted for three years as required by the FDA. There were 97 psychiatrists in 78 institutions and clinics all over the country who participated in this post-marketing surveillance study.

Inclusion criteria were Filipino patients who are $\geq 18$ years, inpatients or outpatients and had met the DSM-IV criteria for schizophrenia. Female subjects must be at least one year postmenopausal, irreversibly surgically sterilized (via hysterectomy, oophorectomy, or bilateral tubal ligation) or abstinent or on effective contraception before entry and throughout the study period.

Exclusion criteria included patients who are on clozapine, any conventional depot neuroleptic or risperidone long acting injection during the last 3 months; with serious unstable medical condition, including recent and present clinically relevant laboratory abnormalities; with history or current symptoms of tardive dyskinesia; history of neuroleptic malignant syndrome; pregnant or breast-feeding female; had participation in an investigational drug trial within 30 days prior to selection; and had known hypersensitivity to paliperidone ER or risperidone.

All patients enrolled in the study signed an informed consent prior to their participation.

\section{Intervention}

The study utilized recommended paliperidone ER starting and maintenance dose at $6 \mathrm{mg} /$ day. However, a flexible dosing in a range of 3 to $12 \mathrm{mg} /$ day was used throughout the study depending on patients needs. Seven (7) tablets of paliperidone ER as starter doses were provided free to patients participating in the study. The remaining doses were purchased by the patients.

\section{Data Collection}

Data were collected using a case-report form filled-up by the investigator based on data availability in the patient records. During the first visit (screening/start of treatment), the subject's demographic data, medical history, previous and current therapy, weight, and overall eligibility of the subject to participate in the trial were determined.

\section{Outcomes Evaluations}

The primary outcome for the study was overall severity of the illness at the initial visit and end visit (visit 2) using the Clinical Global Impression tool (CGI-S). This rating scale was used to rate the severity of a subject's psychotic condition on a 7-point scale ranging from 1 (not ill) to 7 (extremely severe). This measurement permits a global evaluation of the subject's condition at a given time. ${ }^{1}$ The investigator makes an expert clinical global judgment about the severity of the schizophrenia at initial and end visit of each study participants and makes a rating on the CGI form. The CGI-Severity (CGI-S) asks one question: "Considering your total clinical experience with this particular population, how mentally ill is the patient at this time?" which is rated on the following seven-point scale: $1=$ normal, not at all ill; 2=borderline mentally ill; $3=$ mildly ill; $4=$ =moderately ill; $5=$ markedly ill; $6=$ severely ill; $7=$ among the most extremely ill patients. ${ }^{1,14}$ The ratings of the investigators were based upon observed and reported symptoms, behavior, and function in the past seven days. Since symptoms and behavior can swing over a period of one week, the score reflects the average severity level across the seven days.

Safety evaluations included all reported occurrence of events that is related or non-related to Paliperidone intake. Events were assessed and monitored until resolution was noted.

\section{Data Analysis}

Case report forms were checked and encoded using epiinfo version 3.5.1. Epi Info is public domain statistical software for epidemiology developed by Centers for Disease Control and Prevention (CDC) in Atlanta, Georgia (USA). All variables were analyzed and reported using descriptive statistics (mean, standard deviations, frequency and proportions.). 


\section{Results}

\section{Baseline Characteristics}

The mean age of the study cohort $(\mathrm{N}=586)$ was 34.99 ( $\mathrm{SD} \pm 11.10$ ) years. Seventy percent were unmarried and $55 \%$ belonging to reproductive age group of 18 to 35 years. There was a greater number of males (61\%) than females $(39 \%)$. The average weight and height of the study participants were $62.49(\mathrm{SD} \pm 11.10) \mathrm{kg}$ and $162.78(\mathrm{SD} \pm$ 9.62) $\mathrm{cm}$, respectively.

Most of the participants were assessed to be moderately and markedly ill using CGI-S (Table 1).

Table 1. Clinical Global Impression-S

\begin{tabular}{lcccc}
\hline \multicolumn{1}{c}{ Variables } & $\begin{array}{c}\text { Baseline } \\
\mathbf{( N = 5 8 6 )}\end{array}$ & $\begin{array}{c}\text { End Visit } \\
\mathbf{( N = 5 8 6 )}\end{array}$ & $\begin{array}{c}\text { Change } \\
\mathbf{\%}\end{array}$ & p value* $^{*}$ \\
\hline \multicolumn{1}{c}{ Clinical Global } & & & & \\
Impression-S, \% (N) & & & & \\
Normal & $0.2 \%(1)$ & $9 \%(52)$ & +8.2 & $<0.0001^{*}$ \\
Borderline mentally ill & $0.2 \%(1)$ & $23 \%(133)$ & +22.8 & \\
Mildly Ill & $8.2 \%(48)$ & $51 \%(297)$ & +43.0 & \\
Moderate Ill & $36 \%(211)$ & $13 \%(75)$ & -23.0 & \\
Markedly Ill & $39 \%(226)$ & $4 \%(25)$ & -35.0 & \\
Severely Ill & $16 \%(95)$ & $0.7 \%(4)$ & -15.3 & \\
Extremely Ill & $0.7 \%(4)$ & 0 & -0.7 & \\
*Wilcoxon Signed Ranks Test; significant at $\alpha<0.05$ & &
\end{tabular}

Baseline medication dose was at $6 \mathrm{mg}$ for $98 \%$ of the study participants. There was an increase in dose medication from $6 \mathrm{mg}$ to 9 or $12 \mathrm{mg}$ in 27 adults (4\%) while $94 \%$ had remained on $6 \mathrm{mg}$ until the second visit evaluation. Half of the total study participants (46\%) had taken at least one concomitant medication during the study period. The top three concomitant medications taken were biperiden, clonazepam, and diphenhydramine. The incidence density of each drug is $<3$ per 1,000 person-days of follow-up (Table 2).

Table 2. Top Three Concomitant Medications

\begin{tabular}{lcc}
\hline Drug Name & Count & Incidence Density per 1,000 person days \\
\hline Biperiden & 90 & 2.6 \\
Clonazepam & 86 & 2.5 \\
Diphenhydramine & 46 & 1.4 \\
\hline Multiple Response, N=641 (34,072 person/days) \\
$294(45.87 \%)$ patients had taken at least 1 concomitant medication during the study
\end{tabular}

\section{Follow-up and Participation Rates}

A total of 641 Filipino adults were enrolled in the study. Ninety one percent $(\mathrm{N}=586)$ had completed the follow-up into the second or end visit resulting in a 9\% drop out for the duration of the study. Follow-up period had an average of 58.05 (SD \pm 9.36 ) days ranging from a minimum of 4 to a maximum of 98 days. Majority (95.1\%) of the 586 patients was followed up for more than 50 days with a total of 34,072 person-days observation for the entire study duration.

\section{$\underline{\text { Intervention Results }}$}

\section{Primary Outcome}

Clinicians' assessment of the severity of illness showed that majority had shown improvement from their baseline clinical state with the use of Paliperidone ER (Table 1). The proportion of severely ill had decreased by $15 \%$ while the proportion of those who were markedly ill had decreased by $35 \%$ by the end of the second visit.

Overall, after eight weeks of paliperidone treatment, there was a decrease of $58 \%$ in the proportion of study participants who were assessed to be moderately to markedly ill at baseline and an increase of $74 \%$ in the proportion of subjects evaluated to be mildly ill, borderline mentally ill and normal at the end of the second visit. This means that there was a marked improvement in patients observed and reported symptoms, behavior and functions as assessed by physicians using CGI.

\section{Adverse Events}

Fifteen percent of study participants experienced at least one non-serious adverse event during the study. The four most common non-serious adverse events observed include sleeplessness, extrapyramidal symptoms, depression and akathisia. These non-serious adverse events occurred at incidence density of $<2$ events per 1,000 person-days of follow-up (Table 3).

Table 3. Four Most Common Non-Serious Adverse Events

\begin{tabular}{lcc}
\hline Non-Serious Adverse Events & Count & $\begin{array}{c}\text { Incidence Density per } \\
\mathbf{1 , 0 0 0} \text { person days }\end{array}$ \\
\hline Sleeplessness/Insomnia & 47 & 1.3 \\
EPS & 22 & 0.6 \\
Depression & 17 & 0.5 \\
Akathisia & 5 & 0.1 \\
\hline
\end{tabular}

Multiple Response, $N=641$ (34,072 person/days)

$100(15.60 \%)$ patients had experienced at least 1 adverse event during the study

Three cases of serious adverse event involving three study participants were reported and evaluated. One study participant described having swelling of the face, abdominal discomfort and peripheral edema but the episode was assessed not to be drug related. One event was considered as very likely related. The event was described as aggression in a 31-year-old male patient (180 pounds, 69 inches) receiving $6 \mathrm{mg}$ tablet, with a history of alcohol use and smoking. Another possibly related case was reported in 49-year-old female (60 kilograms) with a medical history of seafood allergy, hysterectomy and family history of colon cancer. She experienced nausea, dizziness, poor concentration, severe headache, body malaise, lightheadedness and difficulty in waking up. Treatment with paliperidone ER was discontinued and she eventually reported resolution of symptoms. 


\section{Discussion}

The study was done to evaluate the effect of paliperidone ER among Filipino adult patients diagnosed with schizophrenia. Overall, there was significant improvement among the study participants: improvements in severity, symptoms and functionality. Greater than half of the study participants had improved clinical status after eight weeks of paliperidone ER use.

Clinical improvement in terms of severity of schizophrenia was significant among the study participants: from moderately to severely at baseline to mild illness to normal after two months of medication. This significant improvement in clinical global impression of severity is consistent with the results of previous local studies and foreign studies. ${ }^{15-19}$

Patient compliance to medications is greatly affected by the number of doses taken per day and repeated increases in dosing regimens. Local studies had shown that patients with schizoprenia tend to be shifted and retained on higher maintenance doses with time. In the study conducted by Conde et al. in 2010 among newly diagnosed patients, a total of $22 \%$ of patients were on maintenance of 9 or $12 \mathrm{mg}$ at 3 months. ${ }^{15}$ In this study, the patients enrolled were naïve, and were acutely psychotic with more severe psychopathology (55\% - markedly ill; 23\% - severely ill as measured by CGI-S). Whereas, in this post-marketing surveillance (PMS) study, 39\% were markedly ill and only $16 \%$ were severely ill. Hence, majority of patients in this PMS study were maintained at $6 \mathrm{mg}$ dose.

Fifteen percent of study participants reported at least one adverse event during the study period. However, investigators have commonly classified each as non-serious adverse event. Only three events were considered as serious adverse events. Of these events, only one was considered as very likely and another case was assessed as possibly drugrelated.

All reported non-serious and serious event rates were similar with the results of previous published studies and there are no new safety issues identified with the use of paliperidone ER in this study. ${ }^{20}$

\section{Conclusion}

Paliperidone ER administered at $6 \mathrm{mg}$ single dose improved symptoms and clinical signs among adult Filipinos diagnosed with schizophrenia who are moderately and markedly ill. The drug is well-tolerated, but the dose may need to be increased for more severely ill patients.

Paliperidone ER is one of current useful options for the treatment of patients with schizophrenia.

\section{Declaration of interests}

The study was sponsored by Janssen Pharmaceutical Companies of Johnson and Johnson Philippines. Editorial support for the writing of this manuscript was provided by Noel Juban JR, MD of University of the Philippines-Manila, and this publication was funded by Janssen Pharmaceutical Companies of Johnson and Johnson Philippines. Dr. Erwin Benedicto and Dr. Michael Sionzon are currently connected with Janssen Philippines as the Medical Affairs Manager and Neuroscience Franchise Consultant, respectively.

\section{References}

1. World Health Organization. Mental Health, Disorder Management [Online]. 2006 [cited 2012 June]. Available from http://www.who.int/mental_health/management/schizophrenia/en/

2. World Health Organization-Regional Office for Africa. Mental health Schizophrenia [Online], 2002 [cited 2012 June]. Available form http://www.afro.who.int/mentalhealth/related_diseases/schizophrenia.html

3. Melnik T, Soares BG, Puga ME, Atallah AN. Efficacy and safety of atypical antipsychotic drugs (quetiapine, risperidone, aripiprazole and paliperidone) compared with placebo or typical antipsychotic drugs for treating refractory schizophrenia: overview of systematic reviews. Sao Paulo Med J. 2010; 128(3):141-66

4. United States National Library of Medicine and the National Institutes of Health, Encyclopedia-Schizophrenia [Online]. 2006 [cited 2012 June]. Available from http://www.nlm.nih.gov/medlineplus/ency/article/ 000928.htm

5. Lieberman JA, Stroup TS, McEvoy JP, et al. Effectiveness of antipsychotic drugs in patients with chronic schizophrenia. N Engl J Med. 2005; 353(12):1209-23.

6. Janssen Pharmaceutica. An open-label prospective trial to explore the tolerability, safety and efficacy of flexibly dosed paliperidone ER in subjects with schizophrenia. Clinical Study Report. 2008. Unpublished.

7. Canuso CM, Bossie CA, Turkoz I, Alphs L. Paliperidone extended-release for schizophrenia: effects on symptoms and functioning in acutely ill patients with negative symptoms. Schizophr Res. 2009. 113(1):56-64.

8. Davidson M, Emsley R, Kramer M, et al. Efficacy, safety and early response of paliperidone extended-release tablets (paliperidone ER): results of a 6-week, randomized, placebo-controlled study. Schizophr Res. 2007; 93(1-3):117-30.

9. Kane J, Canas F, Kramer M, et al. Treatment of schizophrenia with paliperidone extended-release tablets: a 6-week placebo-controlled trial. Schizophr Res. 2007; 90(1-3):147-61.

10. Gahr M, Kölle MA, Schönfeldt-Lecuona C, Lepping P, Freudenmann RW. Paliperidone extended-release: does it have a place in antipsychotic therapy? Drug Des Devel Ther. 2011; 5:125-46.

11. Meltzer HY, Bobo WV, Nuamah IF, et al. Efficacy and tolerability of oral paliperidone extended-release tablets in the treatment of acute schizophrenia: pooled data from three 6-week, placebo-controlled studies. J Clin Psychiatry. 2008; 69(5):817-29.

12. Emsley R, Rerwaerts I, Eerdekens M, et al. Efficacy and safety of oral paliperidone extended-release tablets in the treatment of acute schizophrenia: pooled data from three 52-week open-label studies. Int Clin Psychopharmacol. 2008; 23(6): 23:343-56.

13. Nasarallah H, Morosini P, Gagnon DD. Reliability, validity and ability to detect change of the Personal and Social Performance scale in patients with stable schizophrenia. Psychiatry Res. 2008; 161(2):213-24.

14. Busner J, Targum SD. The clinical global impression scale: applying a research tool in clinical practice. Psychiatry. 2007; 4(7):28-37.

15. Conde B, Guiang J, Sionzon M. An open-label prospective trial to explore the tolerability, safety and efficacy of flexibly-dosed paliperidone ER among treatment-naïve and newly-diagnosed patients with schizophrenia. 2010. Unpublished.

16. Schreiner A, Franco M, Buccomino D, et al. Safety, tolerability and efficacy of flexible doses of paliperidone ER in non-acute patients with schizophrenia. Eur Psychiatry. 2009; 24:S1193.

17. Marder SR, Kramer M, Ford L, et al. Efficacy and safety of paliperidone extended-release tablets: results of a 6-week, randomized, placebo-controlled study. Biol Psychiatry. 2007; 62(12):1363-70.

18. Schreiner A, Badescu GM, Jukic V, et al. Safety, tolerability and treatment response of flexible doses of paliperidone ER in acutely exacerbated patients with schizophrenia. Eur Psychiatry. 2009; 24:S1194.

19. Kramer M, Simpsom G, Maciulis V, et al. Paliperidone extended-release tablets for prevention of symptom recurrence in patients with schizophrenia: a randomized, double-blind, placebo-controlled study. J Clin Psychopharmacol. 2007; 27(1):6-14.

20. Clinical Study Report: A Post-Marketing Surveillance Study on the Efficacy and Safety of Paliperidone ER on Filipino Patients. 2010. Unpublished. 